\title{
Sensitivity analyses
}

\begin{tabular}{|c|c|c|c|}
\hline & \multicolumn{3}{|c|}{ Conventional sampling nefrology } \\
\hline Cost unit & & Costs $(€)$ & Total cost \\
\hline \multicolumn{4}{|c|}{ Productivity travel time patient } \\
\hline & Base case & 84 & 259 \\
\hline & Optimistic scenario 1st quartile & 52 & 227 \\
\hline & Pessimistic scenario 3rd quartile & 128 & 303 \\
\hline \multicolumn{4}{|c|}{ Patient costs: travels by public transport } \\
\hline & Base case: car & 23 & 259 \\
\hline & Public transport & 40 & 276 \\
\hline \multicolumn{4}{|c|}{ Productivity loss time in hospital } \\
\hline & Base case & 26 & 259 \\
\hline & Optimistic scenario ready in $25 \mathrm{~min}$ & 15 & 248 \\
\hline & Pessimistic scenario 1 hour waiting & 44 & 277 \\
\hline \multicolumn{4}{|c|}{ Sampling time nurse } \\
\hline & Base case (15 min) & 12 & 259 \\
\hline & Optimistic scenario (sample takes 10 mins) & 8 & 255 \\
\hline & Pessimistic scenario (sample takes 30 mins) & 23 & 271 \\
\hline \multicolumn{4}{|c|}{ Costs of the lab analysis } \\
\hline & Base case & 50 & 259 \\
\hline & CTG tarief & 31 & 241 \\
\hline & Twice base case & 100 & 309 \\
\hline \multicolumn{4}{|c|}{ Costs related to review by pharmacist } \\
\hline & Base case (5 min) & 14 & 259 \\
\hline & Optimistic scenario (2.5 min) & 7 & 252 \\
\hline & Pessimistic scenario (10 min) & 27 & 273 \\
\hline \multicolumn{4}{|c|}{ Time related to contacting patient } \\
\hline & Base case (total $6 \mathrm{~min}$ ) & 16 & 259 \\
\hline & Optimistic scenario (total $3 \mathrm{~min}$ ) & 8 & 251 \\
\hline & Pessimistic scenario (20 min) & 54 & 297 \\
\hline
\end{tabular}

\section{Additional scenario's}

All patient time is 'mantelzorg' time

$\begin{array}{rcc}\text { Base case } & 111 & 259 \\ \text { all time is 'mantelzorg' } & 45 & 193\end{array}$


All patient time is 'productivity loss' = base case

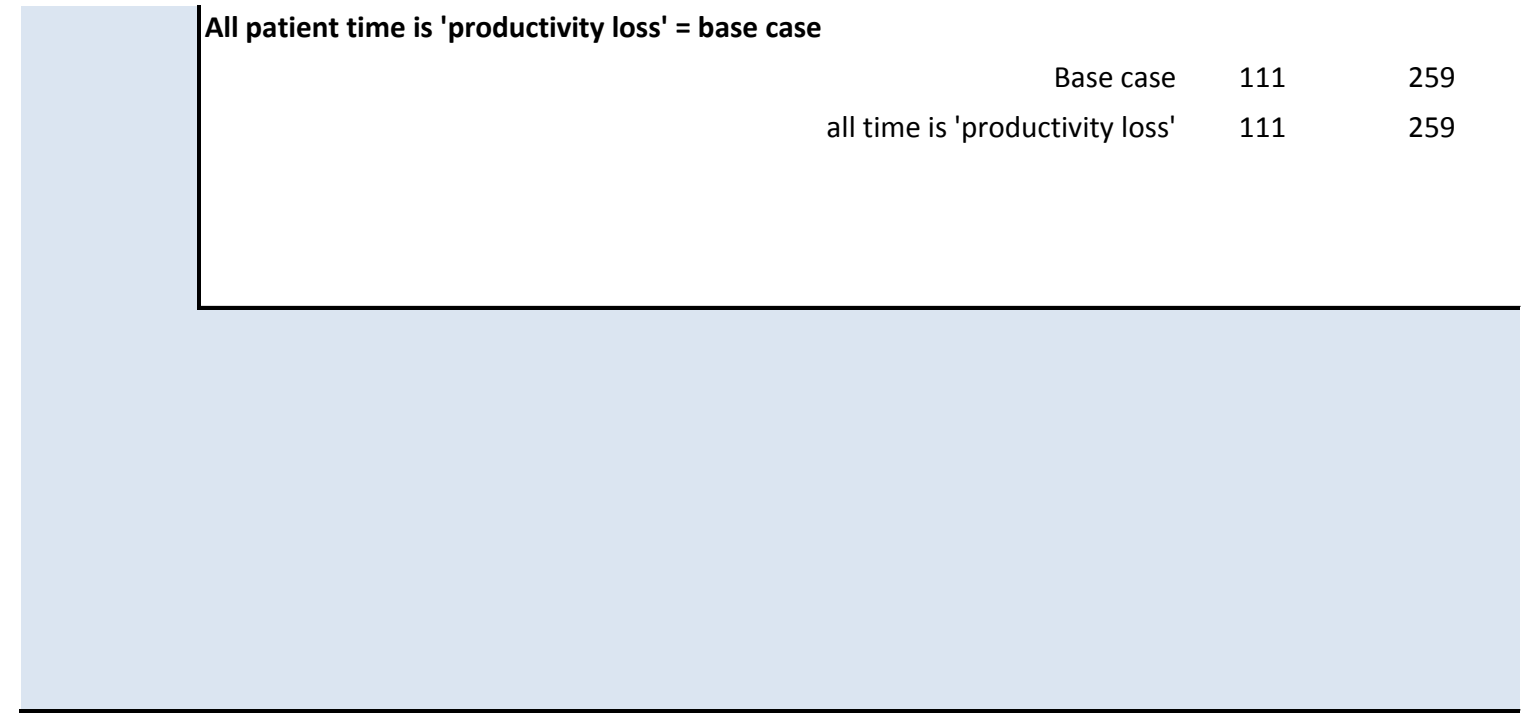




\section{Sensitivity analyses}

\begin{tabular}{|c|c|c|c|}
\hline \multirow[b]{2}{*}{ Difference with base case } & \multicolumn{3}{|c|}{ DBS home sampling nefrology } \\
\hline & & Costs (€) & Total cost \\
\hline \multicolumn{4}{|l|}{ NA } \\
\hline \multicolumn{4}{|l|}{-32} \\
\hline \multicolumn{4}{|l|}{43} \\
\hline \multicolumn{4}{|l|}{ NA } \\
\hline \multicolumn{4}{|l|}{17} \\
\hline \multicolumn{4}{|l|}{ NA } \\
\hline \multicolumn{4}{|l|}{-12} \\
\hline \multicolumn{4}{|l|}{17} \\
\hline \multicolumn{4}{|c|}{ Sampling time parent } \\
\hline NA & Base case (10 min) & 2 & 102 \\
\hline-4 & Optimistic scenario (5 min) & 1 & 101 \\
\hline \multirow[t]{2}{*}{12} & Pessimistic scenario (20 min) & 5 & 105 \\
\hline & Costs of the lab analysis & & \\
\hline NA & Base case & 50 & 102 \\
\hline-19 & CTG tarief & 31 & 84 \\
\hline \multirow[t]{2}{*}{50} & Twice base case & 100 & 152 \\
\hline & Costs related to review by pharmacist & & \\
\hline NA & Base case ( $3 \mathrm{~min})$ & 8 & 102 \\
\hline-7 & Optimistic scenario (2 min) & 5 & 100 \\
\hline \multirow[t]{2}{*}{14} & Pessimistic scenario (10 min) & 27 & 121 \\
\hline & Time related to contacting feed back to patient & & \\
\hline NA & Base case (total $6 \mathrm{~min}$ ) & 16 & 102 \\
\hline-8 & Optimistic scenario (total $3 \mathrm{~min}$ ) & 8 & 94 \\
\hline \multirow[t]{3}{*}{38} & Pessimistic scenario (20 min) & 54 & 141 \\
\hline & Additional scenario's & & \\
\hline & All patient time is 'mantelzorg' time = base case & & \\
\hline NA & Base case & 4 & 102 \\
\hline-66 & all time is 'mantelzorg' & 4 & 102 \\
\hline
\end{tabular}




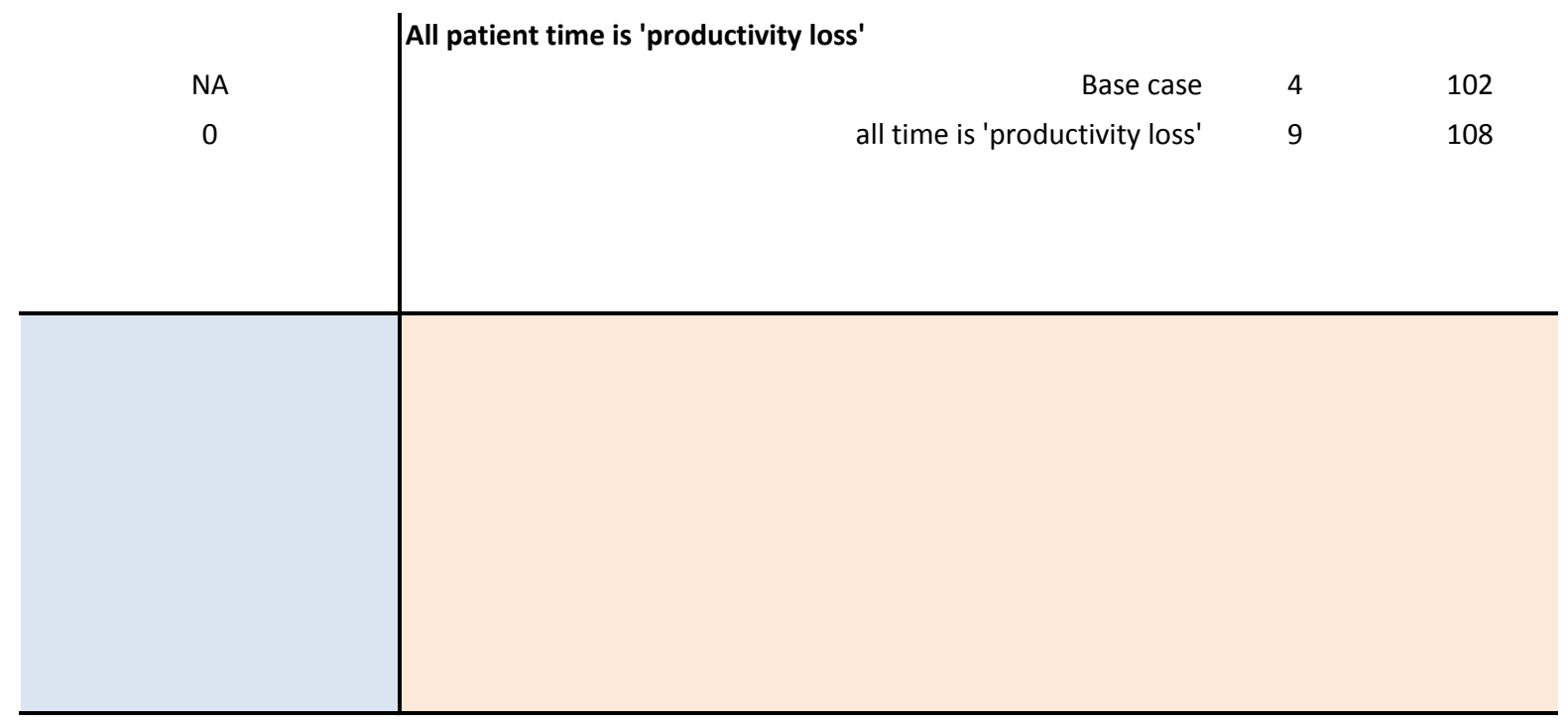


Difference with base case

NA

NA

NA

NA

NA

NA

NA

NA

NA

$-1$

2

NA

$-19$

50

NA

$-3$

19

NA

$-8$

38

NA

0 


\begin{tabular}{c} 
NA \\
6 \\
\\
\hline
\end{tabular}




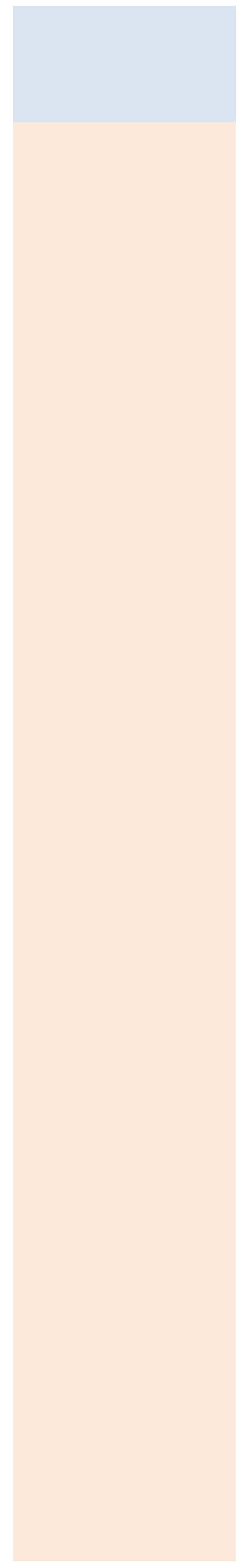




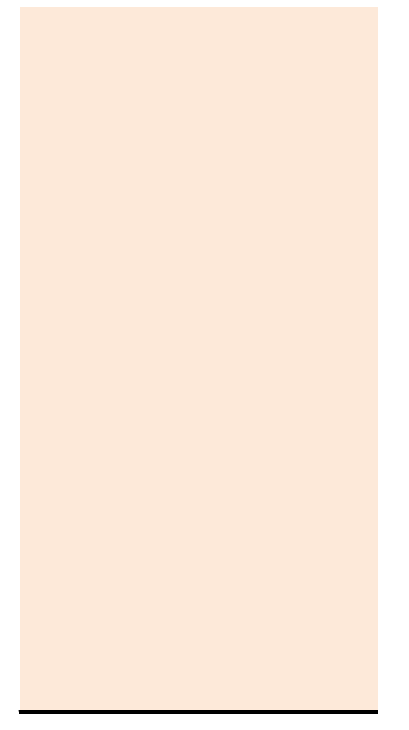

